\title{
Pregnancy Status
}

National Cancer Institute

\section{Source}

National Cancer Institute. Pregnancy Status. NCI Thesaurus. Code C69218.

The pregnancy status of an individual. 